\title{
EVOLUTION ALGEBRAS, AUTOMORPHISMS, AND GRAPHS
}

\author{
ALBERTO ELDUQUE* AND ALICIA LABRA**
}

\begin{abstract}
The affine group scheme of automorphisms of an evolution algebra $\mathcal{E}$ with $\mathcal{E}^{2}=\mathcal{E}$ is shown to lie in an exact sequence $1 \rightarrow \mathbf{D} \rightarrow$ $\operatorname{Aut}(\mathcal{E}) \rightarrow \mathbf{S}$, where $\mathbf{D}$, diagonalizable, and $\mathbf{S}$, constant, depend solely on the directed graph associated to $\mathcal{E}$.

As a consequence, the Lie algebra of derivations $\operatorname{Der}(\mathcal{E})\left(\right.$ with $\mathcal{E}^{2}=\mathcal{E}$ ) is shown to be trivial if the characteristic of the ground field is 0 or 2 , and to be abelian, with a precise description, otherwise.
\end{abstract}

\section{INTRODUCTION}

Evolution algebras were introduced in 2006 by Tian and Vojtechovsky (see [10]) and present many connections with other fields like graph theory, group theory or Markov chains, to mention a few (see Tian's monograph [9]). They have received considerable attention in the last years (see [1] and the references therein).

In this paper, all the algebras considered will be defined over a ground field $\mathbb{F}$, of arbitrary characteristic, and their dimension will be finite. An algebra is just a vector space $\mathcal{A}$ endowed with a bilinear map (the multiplication) $\mathcal{A} \times \mathcal{A} \rightarrow \mathcal{A},(x, y) \mapsto x y$.

Definition 1.1. An evolution algebra is an algebra $\mathcal{E}$ endowed with a basis $B=\left\{v_{1}, v_{2}, \ldots, v_{n}\right\}$, called a natural basis, such that $v_{i} v_{j}=0$ for any $1 \leq i \neq j \leq n$.

Given any evolution algebra $\mathcal{E}$ with natural basis $B=\left\{v_{1}, v_{2}, \ldots, v_{n}\right\}$, and multiplication determined by $v_{i}^{2}=\sum_{i=1}^{n} \alpha_{i j} v_{j}\left(\alpha_{i j} \in \mathbb{F}\right)$, an associated (directed) graph $\Gamma=\Gamma(\mathcal{E}, B)$ is defined in [6]. The set of vertices $V$ of $\Gamma$ is just the natural basis, and the set of edges $E \subseteq V \times V$ consists of those pairs $\left(v_{i}, v_{j}\right)$ with $\alpha_{i j} \neq 0$, that is, $\left(v_{i}, v_{j}\right) \in E$ if $v_{j}$ appears in $v_{i}^{2}$ with nonzero coefficient.

The graph $\Gamma=\Gamma(\mathcal{E}, B)$ is used in [6, 7] to get new results on these algebras and to provide new natural proofs of some known results.

* Supported by grants MTM2017-83506-C2-1-P (AEI/FEDER, UE) and E22_17R (Diputación General de Aragón). Part of this research was done while this author was visiting the Departamento de Matemáticas, Facultad de Ciencias, Universidad de Chile, supported by FONDECYT grant 1170547.

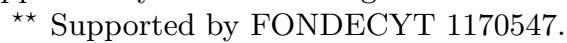


In particular, it is shown in [6] that the group of automorphisms $\operatorname{Aut}(\varepsilon)$ is finite if $\mathcal{E}^{2}=\mathcal{E}$ (or equivalently the matrix $\left(\alpha_{i j}\right)$ is regular). Over an infinite field $\mathbb{F}$, the regular matrices form a Zariski open, and hence dense, set in $\operatorname{Mat}_{n}(\mathbb{F})$. So, in a way, we have that $\operatorname{Aut}(\mathcal{E})$ is finite for "almost all" evolution algebras.

Over fields of positive characteristic, or over nonalgebraically closed fields of characteristic 0, the affine group scheme of automorphisms $\operatorname{Aut}(\mathcal{E})$ contains much more information than $\operatorname{Aut}(\varepsilon)$ including, in particular, the information on the Lie algebra of derivations $\operatorname{Der}(\mathcal{E})$.

Here we follow the functorial approach to affine group schemes (see for instance [1]). An affine group scheme is a representable group-valued functor defined on the category $\operatorname{Alg}_{\mathbb{F}}$ of unital commutative, associative algebras. Thus, given an evolution algebra $\mathcal{E}$, $\operatorname{Aut}(\mathcal{E})$ is the functor $\operatorname{Alg}_{\mathbb{F}} \longrightarrow \operatorname{Grp}$ that takes any object $R$ in $\operatorname{Alg}_{\mathbb{F}}$ to the group $\operatorname{Aut}\left(\mathcal{E}_{R}\right)$ of automorphisms, as an $R$-algebra, of $\mathcal{E}_{R}:=\mathcal{E} \otimes_{\mathbb{F}} R$. The action on morphisms is the natural one.

The Lie algebra $\operatorname{Lie}(\operatorname{Aut}(\mathcal{E}))$ is canonically isomorphic to the Lie algebra of derivations $\operatorname{Der}(\mathcal{E})=\left\{\delta \in \operatorname{End}_{\mathbb{F}}(\varepsilon) \mid \delta(x y)=\delta(x) y+x \delta(y)\right.$ for any $x, y \in \mathcal{E}\}$ (see [5, Example A.43]).

Now, the fact that $\operatorname{Aut}(\mathcal{E})$ is finite if $\mathcal{E}^{2}=\mathcal{E}$ [6. Theorem 4.3] shows, in particular, that $\operatorname{Aut}\left(\mathcal{E}_{\mathbb{F}_{\text {alg }}}\right)$ is finite, where $\mathbb{F}_{\text {alg }}$ is an algebraic closure of $\mathbb{F}$, and hence the affine group scheme $\operatorname{Aut}(\mathcal{E})$ is finite, that is, the Hopf algebra that represents it is finite dimensional.

If the characteristic of the ground field $\mathbb{F}$ is 0 , then any finite affine group scheme is étale, and hence the Lie algebra is trivial. Therefore [6, Theorem 4.8] implies $\operatorname{Der}(\mathcal{E})=0$ if $\mathcal{E}^{2}=\mathcal{E}$ and $\operatorname{char}(\mathbb{F})=0$. (This result over $\mathbb{C}$ has been proven in [2, Theorem 2.6] in a different way). 0 .

However, as some examples in [3] show, this is no longer true if $\operatorname{char}(\mathbb{F})>$

The goal of the present paper is to show that given any evolution algebra $\mathcal{E}$ with $\mathcal{E}^{2}=\mathcal{E}$, there is an exact sequence (7)

$$
1 \longrightarrow \operatorname{Diag}(\Gamma) \longrightarrow \operatorname{Aut}(\mathcal{E}) \longrightarrow \operatorname{Aut}(\Gamma)
$$

where $\operatorname{Aut}(\Gamma)$ is the constant group scheme attached to the group of automorphism of the graph associated to $\mathcal{E}$ in [6], while $\operatorname{Diag}(\Gamma)$ is a finite diagonalizable group scheme defined in terms solely of $\Gamma$. That is the elements in the exact sequence, except $\operatorname{Aut}(\mathcal{E})$ itself, depend only on $\Gamma(! !)$.

An affine group scheme is diagonalizable if it is a subscheme of a torus [11, §2.2] or, equivalently, if the representing Hopf algebra is the gruop algebra of a finitely generated abelian group. In our situation, $\operatorname{Diag}(\Gamma)$ turns out to be a product of schemes of roots of unity $\boldsymbol{\mu}_{N}(N \in \mathbb{N})$, where $\boldsymbol{\mu}_{N}(R)=\left\{r \in R \mid r^{N}=1\right\}$ for any $R$ in $\mathrm{Alg}_{\mathbb{F}}$, which is represented by the quotient $\mathbb{F}[x] /\left(x^{N}-1\right)$, that is, the group algebra of the cyclic group of order $N$. 
On the other hand, given a finite group $G$, the associated constant group scheme $\mathrm{G}$ is the group scheme represented by $\mathbb{F}^{G}:=\operatorname{Maps}(G, \mathbb{F})=\bigoplus_{g \in G} \mathbb{F} \epsilon_{g}$, where

$$
\epsilon_{g}(h)= \begin{cases}1 & \text { if } h=g, \\ 0 & \text { otherwise }\end{cases}
$$

(see [11, §2.4]). For any $R$ in $\operatorname{Alg}_{\mathbb{F}}$ without proper idempotents, $\mathrm{G}(R)$ is (isomorphic to) the group $G$.

Note that $\mathbb{F}^{G} \simeq \mathbb{F} \times \mathbb{F} \times \cdots \times \mathbb{F}$ is the cartesian product of $|G|$ copies of $\mathbb{F}$. In particular, $\mathbb{F}^{G}$ is a separable algebra and hence $G$ is étale.

The paper is structured as follows. Section 2 will be devoted to define and study the diagonalizable affine group scheme $\operatorname{Diag}(\Gamma)$ associated to any graph. For connected $\Gamma, \operatorname{Diag}(\Gamma)$ is either trivial or isomorphic to $\boldsymbol{\mu}_{N}$ for some natural number $N$, given by the so called balance of $\Gamma$. Section 3 will deal with the group of automorphisms of a graph. Its main result: Theorem 3.2, gives the exact sequence (77) mentioned above. This exact sequence induces a short exact sequence (8) which does not split in general. Finally Section 4 is devoted to describe the Lie algebra of derivations of any evolution algebra $\mathcal{E}$ with $\mathcal{E}^{2}=\mathcal{E}$. The description is a direct consequence of our results on the affine group scheme $\operatorname{Aut}(\mathcal{E})$. It turns out that $\operatorname{Der}(\mathcal{E})$ depends only on the graph.

\section{The Diagonal group of A GRAPH}

All the graphs considered in this paper are directed graphs. These are pairs $\Gamma=(V, E)$, consisting of a finite set of vertices $V$ and a set of edges (or arrows) $E \subseteq V \times V$.

Given such a graph, we need some definitions

- A path is a sequence $\gamma=\left(v_{0}, e_{1}, v_{1}, \ldots, v_{n-1}, e_{n}, v_{n}\right)$ where $n \geq 0$, $v_{0}, \ldots, v_{n} \in V, e_{1}, \ldots, e_{n} \in E$, and for each $i=1, \ldots, n$, either $e_{i}=\left(v_{i-1}, v_{i}\right)$ or $e_{i}=\left(v_{i}, v_{i-1}\right)$.

We define the balance of the path $\gamma$ as the integer

$$
\begin{aligned}
\mathrm{b}(\gamma)=\mid\left\{i \mid 1 \leq i \leq n \text { and } e_{i}=(\right. & \left.\left.v_{i-1}, v_{i}\right)\right\} \mid \\
& -\mid\left\{i \mid 1 \leq i \leq n \text { and } e_{i}=\left(v_{i}, v_{i-1}\right)\right\} \mid .
\end{aligned}
$$

that is, $\mathrm{b}(\gamma)$ is obtained by adding 1 if the edge $e_{i}$ goes in the "right" direction (from $v_{0}$ to $v_{n}$ ) and -1 if the edge $e_{i}$ goes in the "wrong" direction, and summing over $i$.

The balance of $\Gamma$ is defined as the greatest common divisor of the absolute values of the balances of the cycles in $\Gamma$ :

$$
\mathrm{b}(\Gamma)=\operatorname{gcd}\{|\mathrm{b}(\gamma)|: \gamma \text { cycle in } \Gamma\} .
$$

- A cycle is a path $\gamma=\left(v_{0}, e_{1}, v_{1}, \ldots, v_{n-1}, e_{n}, v_{n}\right)$ with $v_{0}=v_{n}$. 
- The indegree of a vertex $v$ is the natural number (or 0 )

$$
\operatorname{deg}^{-}(v)=|\{w \in V \mid(w, v) \in E\}|,
$$

while the outdegree is

$$
\operatorname{deg}^{+}(v)=|\{w \in V \mid(v, w) \in E\}| .
$$

The vertex $v$ is said to be a source if $\operatorname{deg}^{-}(v)=0$, and a sink if $\operatorname{deg}^{+}(v)=0$.

- $\Gamma$ is said to be connected if the underlying undirected graph is connected, that is, if for every $v, w \in V$ there exists a path

$$
\gamma=\left(v_{0}, e_{1}, v_{1}, \ldots, v_{n-1}, e_{n}, v_{n}\right)
$$

with $v_{0}=v$ and $v_{n}=w$. Any graph $\Gamma$ is the "disjoint union" of its connected components

Definition 2.1. The diagonal group of a graph $\Gamma=(V, E)$ is the (diagonalizable) affine group scheme $\operatorname{Diag}(\Gamma)$ given by

$$
\operatorname{Diag}(\Gamma)(R)=\left\{\varphi: V \longrightarrow R^{\times} \mid \forall(v, w) \in E, \varphi(w)=\varphi(v)^{2}\right\},
$$

with the natural morphisms.

Note that $\operatorname{Diag}(\Gamma)$ is a subgroup scheme of the torus $\left(\mathbf{G}_{m}\right)^{|V|}$

Let us see a few examples.

\section{Example 2.2.}

$$
\Gamma:
$$

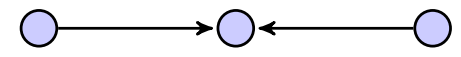

then

$$
\operatorname{Diag}(\Gamma) \simeq\left(\mathbf{G}_{m} \times \mathbf{G}_{m}\right) /\left\{\left(\mu_{1}, \mu_{2}\right) \mid \mu_{1}^{2}=\mu_{2}^{2}\right\} \simeq \mathbf{G}_{m} \times \boldsymbol{\mu}_{2} .
$$

\section{Example 2.3.}

$\Gamma:$

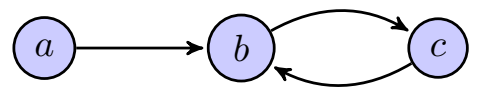

$\Gamma$ has no sinks.

If $\varphi \in \operatorname{Diag}(\Gamma)(R)$ and $\varphi(a)=\mu\left(\in R^{\times}\right)$, then $\varphi(b)=\mu^{2}, \varphi(c)=\mu^{4}$, and $\varphi(b)=\varphi(c)^{2}$, that is $\mu^{2}=\mu^{8}$, so $\mu^{6}=1$. Hence $\operatorname{Diag}(\Gamma) \simeq \boldsymbol{\mu}_{6}$.

\section{Example 2.4.}

$\Gamma:$

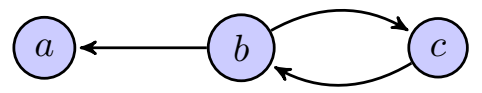

$\Gamma$ has no sources.

Again, if $\varphi \in \operatorname{Diag}(\Gamma)(R)$ and $\varphi(c)=\mu$, then $\varphi(b)=\mu^{2}, \varphi(a)=\mu^{4}$, and $\varphi(c)=\varphi(b)^{2}$, that is, $\mu=\mu^{4}$, so $\mu^{3}=1$. Hence $\operatorname{Diag}(\Gamma) \simeq \boldsymbol{\mu}_{3}$.

From the definitions, we get at once the next result: 
Proposition 2.5. Let $\Gamma=(V, E)$ be a graph with connected components $\Gamma_{i}=\left(V_{i}, E_{i}\right), i=1, \ldots, n$ (so that $V=V_{1} \dot{\cup} \cdots \dot{\cup} V_{n}$ ). Then

$$
\operatorname{Diag}(\Gamma) \simeq \operatorname{Diag}\left(\Gamma_{1}\right) \times \cdots \times \operatorname{Diag}\left(\Gamma_{n}\right) .
$$

If $m=2 s+1$ is an odd natural number the square map

$$
\begin{aligned}
\boldsymbol{\mu}_{m}(R) & \longrightarrow \boldsymbol{\mu}_{m}(R) \\
r & \mapsto r^{2},
\end{aligned}
$$

is a group automorphism for any $R$ in $\operatorname{Alg}_{\mathbb{F}}$, with inverse $r \longrightarrow r^{\frac{1}{2}}:=r^{s+1}$. Therefore, expressions like $r^{2^{-3}}$ make sense: $r^{2^{-3}}=\left(\left(r^{\frac{1}{2}}\right)^{\frac{1}{2}}\right)^{\frac{1}{2}}$.

Lemma 2.6. Let $\Gamma=(V, E)$ be a graph, $\gamma=\left(v_{0}, e_{1}, v_{1}, \ldots, v_{n-1}, e_{n}, v_{n}\right)$ be a path in $\Gamma$. Let $\varphi \in \operatorname{Diag}(\Gamma)(R)$ for $R$ in $\operatorname{Alg}_{\mathbb{F}}$, such that $\varphi\left(v_{i}\right) \in \boldsymbol{\mu}_{m_{i}}(R)$ with $m_{i}$ odd for any $i=0, \ldots, n$. Then $\varphi\left(v_{n}\right)=\varphi\left(v_{0}\right)^{2^{\mathrm{b}(\gamma)}}$.

Proof. Imagine that $\gamma=\left(v_{0}, e_{1}, v_{1}, e_{2}, v_{2}, e_{3}, v_{3}\right)$ with $e_{1}=\left(v_{1}, v_{0}\right), e_{2}=$ $\left(v_{1}, v_{2}\right)$, and $e_{3}=\left(v_{3}, v_{2}\right)$, so $\mathrm{b}(\gamma)=-1$.

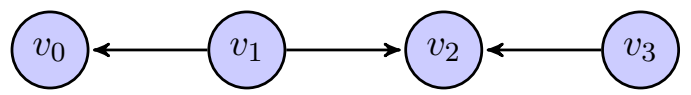

Then

- As $e_{1}=\left(v_{1}, v_{0}\right) \in E, \varphi\left(v_{0}\right)=\varphi\left(v_{1}\right)^{2}$, so $\varphi\left(v_{1}\right)=\varphi\left(v_{0}\right)^{\frac{1}{2}}=\varphi\left(v_{0}\right)^{2^{-1}}$.

- As $e_{2}=\left(v_{1}, v_{2}\right) \in E, \varphi\left(v_{2}\right)=\varphi\left(v_{1}\right)^{2}$, so $\varphi\left(v_{2}\right)=\left(\varphi\left(v_{0}\right)^{2^{-1}}\right)^{2}=$ $\varphi\left(v_{0}\right)$.

- As $e_{3}=\left(v_{3}, v_{2}\right) \in E, \varphi\left(v_{2}\right)=\varphi\left(v_{3}\right)^{2}$, so $\varphi\left(v_{3}\right)=\varphi\left(v_{2}\right)^{-1}=$ $\varphi\left(v_{1}\right)^{2^{-1}}=\varphi\left(v_{0}\right)^{2^{\mathrm{b}(\gamma)}}$.

The general argument follows the same lines.

Our next result determines the diagonal group of connected graphs without sources. Note that the graphs attached to evolution algebras $\mathcal{E}$ with $\varepsilon^{2}=\mathcal{E}$ have no sources.

Theorem 2.7. Let $\Gamma=(V, E)$ be a connected graph with no sources. Then $\operatorname{Diag}(\Gamma) \simeq \boldsymbol{\mu}_{N}$ where $N=2^{\mathrm{b}(\Gamma)}-1$.

Proof. First, the arguments in the proof of [6, Theorem 4.8] show that for any $R$ in $\operatorname{Alg}_{\mathbb{F}}$, any $\varphi \in \operatorname{Diag}(\Gamma)(R)$, and any vector $v \in V, \varphi(v) \in \boldsymbol{\mu}_{2^{s}-1}(R)$ for some natural number $s$.

Fix a vertex $a \in V$, and consider the restriction homomorphism

$$
\begin{aligned}
\Phi_{a}: \operatorname{Diag}(\Gamma) & \longrightarrow \mathbf{G}_{m} \\
\varphi & \mapsto \varphi(a) .
\end{aligned}
$$

We will follow several steps:

- $\Phi_{a}$ is one-to-one.

Actually, for $R$ in $\operatorname{Alg}_{\mathbb{F}}$, and $\varphi \in \operatorname{Diag}(\Gamma)(R)$, with $\varphi(a)=$ 1 , by connectedness for any vertex $v \in V$ there is a path $\gamma=$ 
$\left(v_{0}, e_{1}, v_{1}, \ldots, v_{n-1}, e_{n}, v_{n}\right)$ with $v_{0}=a$ and $v_{n}=v$. By Lemma 2.6, $\varphi(v)=\varphi(a)^{2^{\mathrm{b}(\gamma)}}=1^{2^{\mathrm{b}(\gamma)}}=1$.

- For any $R$ in $\operatorname{Alg}_{\mathbb{F}}$, and $\varphi \in \operatorname{Diag}(\Gamma)(R), \varphi(a) \in \boldsymbol{\mu}_{N}(R)$.

Indeed, by the previous argument, for any $v \in V, \varphi(v)=\varphi(a)^{2^{\mathrm{b}(\gamma)}}$ for any path $\gamma$ connecting $a$ and $v$. As the order of $\varphi(a)$ is odd, $\varphi(a)$ and $\varphi(v)$ generate the same subgroup of $R^{\times}$. In particular $\varphi(a)$ and $\varphi(v)$ have the same order.

Given any cycle $\gamma=\left(v_{0}, e_{1}, v_{1}, \ldots, v_{n-1}, e_{n}, v_{n}\right)$ in $\Gamma\left(v_{n}=v_{0}\right)$, we get $\varphi\left(v_{0}\right)=\varphi\left(v_{0}\right)^{2^{\mathrm{b}(\gamma)}}$, or $\varphi\left(v_{0}\right)^{2^{\mathrm{b}(\gamma)}-1}=1$. Thus the order of $\varphi(a)$ divides $2^{|\mathrm{b}(\gamma)|}-1$ for any cycle $\gamma$. Using that $2^{\operatorname{gcd}\left(m_{1}, m_{2}\right)}-1=$ $\operatorname{gcd}\left(2^{m_{1}}-1,2^{m_{2}}-1\right)$, our result follows.

- The image of $\Phi_{a}$ is exactly $\boldsymbol{\mu}_{N}$.

For any $R$ in $\operatorname{Alg}_{\mathbb{F}}$, and any $\mu \in \mu_{N}(R)$, define $\varphi: V \longrightarrow R^{\times}$as follows: For any $v \in V$, select a path connecting $a$ and $v: \gamma=$ $\left(v_{0}, e_{1}, v_{1}, \ldots, v_{n-1}, e_{n}, v_{n}\right)$ with $v_{0}=a$ and $v_{n}=v$, and define $\varphi(v)=\mu^{2^{\mathrm{b}(\gamma)}}$. This is well defined, because for any other path $\hat{\gamma}=\left(\hat{v}_{0}, \hat{e}_{1}, \hat{v}_{1}, \ldots, \hat{v}_{n-1}, \hat{e}_{n}, \hat{v}_{n}\right)$ connecting $a=\hat{v}_{0}$ and $v=\hat{v}_{n}$, then $\gamma \hat{\gamma}^{-1}:=\left(v_{0}, e_{1}, v_{1}, \ldots, v_{n-1}, e_{n}, v_{n}=\hat{v}_{n}, \hat{e}_{n}, \hat{v}_{n-1}, \ldots, \hat{e}_{1}, \hat{v}_{0}\right)$

is a cycle with balance $\mathrm{b}\left(\gamma \hat{\gamma}^{-1}\right)=\mathrm{b}(\gamma)-\mathrm{b}(\hat{\gamma})$ and, therefore, $\mu=$ $\mu^{2^{\mathrm{b}(\gamma)-\mathrm{b}(\hat{\gamma})} \text {. Hence }}$

$$
\mu^{2^{\mathrm{b}(\hat{\gamma})}}=\left(\mu^{2^{\mathrm{b}(\gamma)-\mathrm{b}(\hat{\gamma})}}\right)^{2^{\mathrm{b}(\hat{\gamma})}}=\mu^{2^{\mathrm{b}(\gamma)}} .
$$

Finally, $\varphi \in \operatorname{Diag}(\Gamma)(R)$, because for any $e=(v, w) \in E$, if $\gamma=$ $\left(v_{0}, e_{1}, v_{1}, \ldots, v_{n-1}, e_{n}, v_{n}\right)$ is a path connecting $a=v_{0}$ and $v=v_{n}$, then $\gamma^{\prime}=\left(v_{0}, e_{1}, v_{1}, \ldots, v_{n-1}, e_{n}, v_{n}, e, w\right)$ is a path connecting $a$ and $w$ with $\mathrm{b}\left(\gamma^{\prime}\right)=\mathrm{b}(\gamma)+1$. Hence, $\varphi(w)=\mu^{2^{\mathrm{b}\left(\gamma^{\prime}\right)}}=\left(\mu^{2^{\mathrm{b}(\gamma)}}\right)^{2}=\varphi(v)^{2}$.

Corollary 2.8. Let $\Gamma=(V, E)$ be a connected graph with no sources and with a loop $e=(v, v)$. Then $\operatorname{Diag}(\Gamma)=1$.

Let $\mathcal{E}$ be an evolution algebra with natural basis $B=\left\{v_{1}, \ldots, v_{n}\right\}$ and let $\Gamma=\Gamma(\mathcal{E}, B)=(V, E)$ be the attached graph $(V=B)$. For any $R$ in $A_{\lg _{\mathbb{F}}}$, and any $\varphi \in \operatorname{Diag}(\Gamma)(R), \varphi$ induces the linear (diagonal) isomorphism

$$
\begin{aligned}
\hat{\varphi}: \mathcal{E}_{R} & \longrightarrow \mathcal{E}_{R} \\
v_{i} & \mapsto \varphi\left(v_{i}\right) v_{i} .
\end{aligned}
$$

Let $v_{i}^{2}=\sum_{j=1}^{n} \alpha_{i j} v_{j}$ for $i=1, \ldots, n$, with $\alpha_{i j} \in \mathbb{F}$, then

$$
\hat{\varphi}\left(v_{i}^{2}\right)=\sum_{j=1}^{n} \alpha_{i j} \hat{\varphi}\left(v_{j}\right)=\sum_{j=1}^{n} \alpha_{i j} \varphi\left(v_{j}\right) v_{j},
$$

and $\hat{\varphi}\left(v_{i}\right)^{2}=\varphi\left(v_{i}\right)^{2} \sum_{j=1}^{n} \alpha_{i j} v_{j}$ 
But if $\alpha_{i j} \neq 0$, then $\left(v_{i}, v_{j}\right) \in E$, so $\varphi\left(v_{j}\right)=\varphi\left(v_{i}\right)^{2}$. Hence $\hat{\varphi} \in \operatorname{Aut}\left(\mathcal{E}_{R}\right)$ and we obtain the following result:

Theorem 2.9. Let $\mathcal{E}$ be an evolution algebra with natural basis $B$ and let $\Gamma=\Gamma(\mathcal{E}, B)$ be the attached graph. Then there is an injective homomorphism $\iota: \operatorname{Diag}(\Gamma) \longrightarrow \operatorname{Aut}(\mathcal{E})$ such that for any $R$ in $\operatorname{Alg}_{\mathbb{F}}$, and any $R$-point $\varphi \in \operatorname{Diag}(\Gamma)(R), \iota(\varphi)=\hat{\varphi}$ (as in (1)).

\section{Graph Automorphisms}

The goal of this section is, given an evolution algebra $\mathcal{E}$ with $\mathcal{E}^{2}=\mathcal{E}$ with attached graph $\Gamma(\mathcal{E}, B)$ (which is independent, up to isomorphism, of the natural basis $B$ chosen [6, Corollary 4.7]), to show the existence of a natural homomorphism

$$
\rho: \operatorname{Aut}(\mathcal{E}) \longrightarrow \operatorname{Aut}(\Gamma)
$$

where $\operatorname{Aut}(\Gamma)$ is the constant group scheme attached to the group of automorphisms of $\Gamma$, denoted by $\operatorname{Aut}(\Gamma)$. If $B=\left\{v_{1}, \ldots, v_{n}\right\}$ is a natural basis we may identify $\operatorname{Aut}(\Gamma)$ with a subgroup of the symmetric group $S_{n}$ of degree $n$ :

$$
\operatorname{Aut}(\Gamma) \simeq\left\{\sigma \in S_{n} \mid \forall 1 \leq i, j \leq n,\left(v_{i}, v_{j}\right) \in E \Rightarrow\left(v_{\sigma(i)}, v_{\sigma(j)}\right) \in E\right\} .
$$

If we just look at the rational points in $\operatorname{Aut}(\mathcal{E})=\operatorname{Aut}(\mathcal{E})(\mathbb{F})$, any $\varphi \in \operatorname{Aut}(\mathcal{E})$ has an attached permutation $\sigma \in \operatorname{Aut}(\Gamma)$ such that $\varphi\left(v_{i}\right) \in \mathbb{F}^{\times} v_{\sigma(i)}$ for any $i=1, \ldots, n$ ([6, Theorem 4.4]). Thus the coordinate matrix of $\varphi$ relative to $B$ is a monomial matrix (i.e., it has exactly one nonzero entry in each row and column). In order to deal with the group scheme $\operatorname{Aut}(\mathcal{E})$, some extra care must be taken. Let $R$ be in $\operatorname{Alg}_{\mathbb{F}}$, and let $\varphi \in \operatorname{Aut}(\mathcal{E})(R)=\operatorname{Aut}\left(\varepsilon_{R}\right)$, with $\varphi\left(v_{i}\right)=\sum_{j=i}^{n} r_{i j} v_{j}$ for any $i=1, \ldots, n$. Then $r=\operatorname{det}\left(r_{i j}\right) \in R^{\times}$:

$$
r=\sum_{\sigma \in S_{n}}(-1)^{\sigma} r_{\sigma(1) 1} \cdots r_{\sigma(n) n} \in R^{\times} .
$$

For any $i \neq j$ we have $0=\varphi\left(v_{i} v_{j}\right)=\varphi\left(v_{i}\right) \varphi\left(v_{j}\right)=\sum_{k=1}^{n} r_{i k} r_{j k} v_{k}^{2}$.

Because $\mathcal{E}^{2}=\mathcal{E},\left\{v_{1}^{2}, \ldots, v_{n}^{2}\right\}$ form a basis of $\mathcal{E}$ and hence

$$
r_{i k} r_{j k}=0 \text { for any } 1 \leq i, j \leq n \text { with } i \neq j \text {. }
$$

Therefore, for any $\sigma \neq \tau$ in $S_{n},\left(r_{\sigma(1) 1} \cdots r_{\sigma(n) n}\right)\left(r_{\tau(1) 1} \cdots r_{\tau(n) n}\right)=0$.

For any $\sigma \in S_{n}$, consider the element

$$
e_{\sigma}^{\varphi}=(-1)^{\sigma} r^{-1} r_{\sigma(1) 1} \cdots r_{\sigma(n) n}
$$

Then $1=\sum_{\sigma \in S_{n}} e_{\sigma}^{\varphi}$, and $e_{\sigma}^{\varphi} e_{\tau}^{\varphi}=0$ for $\sigma \neq \tau$ in $S_{n}$. Therefore, the $e_{\sigma}^{\varphi}$ 's are orthogonal idempotent elements, and $R=\bigoplus_{\sigma \in S_{n}} R e_{\sigma}^{\varphi}$. Moreover, (3) implies

$$
r_{i j} e_{\sigma}^{\varphi}=0 \quad \text { unless } i=\sigma(j) \text {, }
$$


and the coordinate matrix $\left(r_{i j}\right)$ of $\varphi$ splits into a sum of monomial matrices over the orthogonal ideals $\operatorname{Re}_{\sigma}^{\varphi}$. Thus, for instance, with $n=3$ we have:

$$
\begin{aligned}
& e_{1}^{\varphi}=r_{11} r_{22} r_{33}, \quad e_{(123)}^{\varphi}=r_{21} r_{32} r_{13}, \quad e_{(132)}^{\varphi}=r_{31} r_{12} r_{23}, \\
& e_{(12)}^{\varphi}=-r_{21} r_{12} r_{33}, \quad e_{(23)}^{\varphi}=-r_{11} r_{32} r_{23}, \quad e_{(13)}^{\varphi}=-r_{31} r_{22} r_{13} \text {. }
\end{aligned}
$$

and $A=\left(r_{i j}\right)=\sum_{\sigma \in S_{3}} A_{\sigma}$, with $A_{\sigma}=e_{\sigma}^{\varphi} A \in \operatorname{Mat}_{3}\left(\operatorname{Re}_{\sigma}^{\varphi}\right)$ a monomial matrix thanks to (4):

$$
\begin{aligned}
A_{1} & =e_{1}^{\varphi}\left(\begin{array}{ccc}
r_{11} & 0 & 0 \\
0 & r_{22} & 0 \\
0 & 0 & r_{33}
\end{array}\right), & & A_{(123)}=e_{(123)}^{\varphi}\left(\begin{array}{ccc}
0 & 0 & r_{13} \\
r_{21} & 0 & 0 \\
0 & r_{32} & 0
\end{array}\right), \\
A_{(132)} & =e_{(132)}^{\varphi}\left(\begin{array}{ccc}
0 & r_{12} & 0 \\
0 & 0 & r_{23} \\
r_{13} & 0 & 0
\end{array}\right), & & A_{(12)}=e_{(12)}^{\varphi}\left(\begin{array}{ccc}
0 & r_{12} & 0 \\
r_{21} & 0 & 0 \\
0 & 0 & r_{33}
\end{array}\right), \\
A_{(23)} & =e_{(23)}^{\varphi}\left(\begin{array}{ccc}
r_{11} & 0 & 0 \\
0 & 0 & r_{23} \\
0 & r_{32} & 0
\end{array}\right), & & A_{(13)}=e_{(13)}^{\varphi}\left(\begin{array}{ccc}
0 & 0 & r_{13} \\
0 & r_{22} & 0 \\
r_{31} & 0 & 0
\end{array}\right) .
\end{aligned}
$$

Moreover, if $\sigma \in S_{n}$ and $e_{\sigma}^{\varphi} \neq 0$, then the monomial matrix

$$
A_{\sigma}=e_{\sigma}^{\varphi}\left(r_{i j}\right)=\sum_{i=1}^{n} e_{\sigma}^{\varphi} r_{\sigma(i) i} E_{\sigma(i) i},
$$

where $E_{i j}$ denotes the matrix with 1 in the $(i j)$ slot and 0 's elsewhere, correspond to an automorphism of $\mathcal{E}_{R e_{\sigma}^{\varphi}}$. This forces $\sigma \in \operatorname{Aut}(\Gamma)$. Therefore,

$$
e_{\sigma}^{\varphi} \neq 0 \text { only if } \sigma \in \operatorname{Aut}(\Gamma), \quad 1=\sum_{\sigma \in \operatorname{Aut}(\Gamma)} e_{\sigma}^{\varphi} .
$$

Recall that the coordinate Hopf algebra of the constant group scheme $\operatorname{Aut}(\Gamma)$ is $\mathbb{F}^{\operatorname{Aut}(\Gamma)}=\operatorname{Maps}(\operatorname{Aut}(\Gamma), \mathbb{F})$, which has a natural basis $\left\{\epsilon_{\sigma} \mid \sigma \in\right.$ $\operatorname{Aut}(\Gamma)\}$, with

$$
\epsilon_{\sigma}(\tau)= \begin{cases}1 & \text { if } \sigma=\tau \\ 0 & \text { otherwise }\end{cases}
$$

Then $\operatorname{Aut}(\Gamma)(R)$ is identified with $\operatorname{Hom}_{\operatorname{Alg}_{\mathbb{F}}}\left(\mathbb{F}^{\operatorname{Aut}(\Gamma)}, R\right)$.

We are ready to define the homomorphism $\rho$ in (2). For $R$ in $\operatorname{Alg}_{\mathbb{F}}$ and $\varphi \in \operatorname{Aut}(\mathcal{E})(R)=\operatorname{Aut}\left(\mathcal{E}_{R}\right)$, the image of $\varphi$ under $\rho$ is defined as the element $\rho(\varphi) \in \operatorname{Hom}_{\mathrm{Alg}_{\mathbb{F}}}\left(\mathbb{F}^{\mathrm{Aut}(\Gamma)}, R\right)$ given by

$$
\begin{aligned}
\rho(\varphi): \mathbb{F}^{\operatorname{Aut}(\Gamma)} & \longrightarrow R \\
\epsilon_{\sigma} & \mapsto e_{\sigma}^{\varphi} .
\end{aligned}
$$

It is trivially checked that this gives a homomorphism $\rho: \operatorname{Aut}(\mathcal{E}) \rightarrow$ Aut $(\Gamma)$. 
Remark 3.1. Exactly as over $\mathbb{F}$, if $R$ in $\operatorname{Alg}_{\mathbb{F}}$ has no proper idempotents, then $1=e_{\sigma}^{\varphi}$ for a unique $\sigma \in \operatorname{Aut}(\Gamma)$ and the matrix of $\varphi$ is a monomial matrix attached to $\sigma$. In this case $\operatorname{Aut}(\Gamma)(R) \simeq \operatorname{Aut}(\Gamma)$ and $\rho(\varphi)$ is just $\sigma$ under this identification.

The main result of this section is the following:

Theorem 3.2. Let $\mathcal{E}$ be an evolution algebra with $\mathcal{E}^{2}=\mathcal{E}$ and natural basis $B=\left\{v_{1}, \ldots, v_{n}\right\}$. Let $\Gamma=\Gamma(\mathcal{E}, B)$ be its associated graph. Then the sequence

$$
1 \longrightarrow \operatorname{Diag}(\Gamma) \stackrel{\iota}{\longrightarrow} \operatorname{Aut}(\mathcal{E}) \stackrel{\rho}{\longrightarrow} \operatorname{Aut}(\Gamma)
$$

is exact.

Proof. $\operatorname{ker}(\rho)(R)$ consists of the automorphisms $\varphi \in \operatorname{Aut}(\mathcal{E})(R)=\operatorname{Aut}\left(\mathcal{E}_{R}\right)$ such that $e_{\sigma}^{\varphi}=0$ for any $1 \neq \sigma \in \operatorname{Aut}(\Gamma)$. Hence $1=e_{1}^{\varphi}$ and $\varphi$ is diagonal, that is, the elements of $B$ are eigenvectors for $\varphi$. These automorphisms are precisely the elements in the image of $\iota$.

Example 3.3. The homomorphism $\rho$ is not surjective in general. Take, for instance, the evolution algebra $\mathcal{E}=\mathbb{F} v_{1} \oplus \mathbb{F} v_{2}$, with natural basis $B=$ $\left\{v_{1}, v_{2}\right\}$, and multiplication given by $v_{1}^{2}=v_{1}+\alpha v_{2}, v_{2}^{2}=\beta v_{1}+v_{2}$, with $0 \neq \alpha, \beta \in \mathbb{F}, \alpha \neq \beta, \alpha \beta \neq 1$. Then the associated graph $\Gamma(\mathcal{E}, B)$ is the complete graph

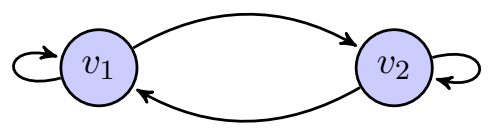

While $\operatorname{Aut}(\Gamma)=C_{2}$, let us check that $\operatorname{Aut}(\mathcal{E})=1$. To do that, it is enough to prove that $\operatorname{Aut}\left(\mathcal{E}_{R}\right)=1$ for $R$ in $\operatorname{Alg}_{\mathbb{F}}$ without proper idempotents.

The arguments above show that the coordinate matrix relative to $B=$ $\left\{v_{1}, v_{2}\right\}$ of any $\varphi \in \operatorname{Aut}\left(\mathcal{E}_{R}\right)$ is either

$$
\left(\begin{array}{cc}
r_{1} & 0 \\
0 & r_{2}
\end{array}\right) \quad \text { or } \quad\left(\begin{array}{cc}
0 & r_{1} \\
r_{2} & 0
\end{array}\right)
$$

with $r_{1}, r_{2} \in R^{\times}$.

In the first case $\varphi\left(v_{1}^{2}\right)=r_{1} v_{1}+\alpha r_{2} v_{2}$, while $\varphi\left(v_{1}\right)^{2}=r_{1}^{2}\left(v_{1}+\alpha v_{2}\right)$, so $r_{1}^{2}=r_{1}=r_{2}$, and hence, due to the absence of proper idempotents, $\varphi=\mathrm{id}$.

In the second case $\varphi\left(v_{1}^{2}\right)=r_{1} v_{2}+\alpha r_{2} v_{1}$, while $\varphi\left(v_{1}\right)^{2}=r_{1}^{2} v_{2}^{2}=r_{1}^{2}\left(\beta v_{1}+\right.$ $\left.v_{2}\right)$, so $r_{1}^{2}=r_{1}$ and $\alpha r_{2}=\beta r_{1}^{2}$. Hence, $r_{1}=1, r_{2}=\beta \alpha^{-1} \neq 1$. But $\varphi\left(v_{2}^{2}\right)=\varphi\left(v_{2}\right)^{2}$ forces $r_{2}=1$, a contradiction.

Any subgroup scheme of a constant group scheme is itself a constant group scheme. Hence we have the next consequence:

Corollary 3.4. Let $\mathcal{E}$ be an evolution algebra with $\mathcal{E}^{2}=\mathcal{E}$ and natural basis $B=\left\{v_{1}, \ldots, v_{n}\right\}$. Let $\Gamma=\Gamma(\mathcal{E}, B)$ be its associated graph. Then there is a subgroup $H$ of $\operatorname{Aut}(\Gamma)$ and a short exact sequence

$$
1 \longrightarrow \operatorname{Diag}(\Gamma) \stackrel{\iota}{\longrightarrow} \operatorname{Aut}(\mathcal{E}) \stackrel{\rho}{\longrightarrow} \mathrm{H} \longrightarrow 1,
$$


where $\mathrm{H}$ is the constant group scheme associated to $H$.

Example 3.5. The short exact sequence in Corollary 3.4 does not split in general. Take, for instance the evolution algebra $\mathcal{E}=\mathbb{F} v_{1} \oplus \mathbb{F} v_{2}$ with $v_{1}^{2}=v_{2}$, $v_{2}^{2}=\alpha v_{1}$, with $0 \neq \alpha \in \mathbb{F}$. The associated graph is

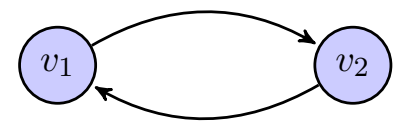

Then $\operatorname{Diag}(\Gamma)=\boldsymbol{\mu}_{3}$ (Theorem 2.7) and $\rho: \operatorname{Aut}(\mathcal{E}) \longrightarrow \operatorname{Aut}(\Gamma) \simeq \mathrm{C}_{2}$ is surjective, as it is so over an algebraic closure $\mathbb{F}_{\text {alg. }}$. Indeed, over $\mathbb{F}_{\text {alg }}$ the assignment

$$
v_{1} \mapsto \alpha^{-1 / 3} v_{2}, \quad v_{2} \mapsto \alpha^{1 / 3} v_{1},
$$

gives an automorphism $\varphi$ with $\rho(\varphi)$ being the generator of $\operatorname{Aut}(\Gamma)$. Moreover, $\varphi^{2}=\mathrm{id}$ and this proves that (8) splits over $\mathbb{F}_{\text {alg }}$.

Let us check that the short exact sequence

$$
1 \longrightarrow \operatorname{Diag}(\Gamma) \longrightarrow \operatorname{Aut}(\varepsilon) \longrightarrow \operatorname{Aut}(\Gamma) \longrightarrow 1
$$

splits if and only if there is $\mu \in \mathbb{F}$ such that $\alpha=\mu^{3}$.

Actually, if $\alpha=\mu^{3}$ the assignment (9) makes sense over $\mathbb{F}$, so the sequence splits. Conversely, if (10) splits, there is an automorphism $\varphi \in \operatorname{Aut}(\mathcal{E})$ with $\varphi^{2}=$ id, such that $\varphi\left(v_{1}\right) \in \mathbb{F}^{\times} v_{2}, \varphi\left(v_{2}\right) \in \mathbb{F}^{\times} v_{1}$. With $\varphi\left(v_{1}\right)=\nu v_{2}$, $\varphi\left(v_{2}\right)=\mu v_{1}$, we get $\nu=\mu^{-1}$, as $\varphi^{2}=\mathrm{id}$, and

$$
\mu v_{1}=\varphi\left(v_{2}\right)=\varphi\left(v_{1}^{2}\right)=\varphi\left(v_{1}\right)^{2}=\mu^{-2} v_{2}^{2}=\mu^{-2} \alpha v_{1},
$$

so that $\alpha=\mu^{3}$.

\section{Derivations}

The results of the previous sections allow us to compute easily the Lie algebra of derivations of any evolution algebra $\mathcal{E}$, with $\mathcal{E}^{2}=\mathcal{E}$. This Lie algebra depends only on the associated graph!

Theorem 4.1. Let $\mathcal{E}$ be an evolution algebra with $\mathcal{E}^{2}=\mathcal{E}$. Let $B$ be a natural basis and let $\Gamma=\Gamma(\mathcal{E}, B)$ be the attached graph. Then:

(1) If the characteristic of $\mathbb{F}$ is 0 or 2 , then $\operatorname{Der}(\mathcal{E})=0$.

(2) If the characteristic of $\mathbb{F}$ is $p \neq 0,2$, then $\operatorname{Der}(\mathcal{E})$ is an abelian Lie algebra whose dimension is the number of connected components $\Gamma_{i}$ of $\Gamma$ such that the order of 2 in $\mathbb{Z} / p \mathbb{Z}$ divides the balance $\mathrm{b}\left(\Gamma_{i}\right)$.

Proof. The exact sequence (7) induces an exact sequence (see eq. [Milne, 10d]):

$$
0 \longrightarrow \operatorname{Lie}(\operatorname{Diag}(\Gamma)) \stackrel{\mathrm{d} \iota}{\longrightarrow} \operatorname{Lie}(\operatorname{Aut}(\mathcal{E})) \stackrel{\mathrm{d} \rho}{\longrightarrow} \operatorname{Lie}(\operatorname{Aut}(\Gamma))
$$

$\operatorname{But} \operatorname{Lie}(\operatorname{Aut}(\Gamma))=0$, as $\operatorname{Aut}(\Gamma)$ is a constant group scheme, and hence étale. On the other hand, Lie $(\operatorname{Aut}(\mathcal{E}))=\operatorname{Der}(\mathcal{E})$ (see [5, Example A.43]), so that $\operatorname{Der}(\mathcal{E})$ is isomorphic to $\operatorname{Lie}(\operatorname{Diag}(\Gamma))$ through the differential of $\iota$. 
However, $\operatorname{Lie}\left(\boldsymbol{\mu}_{m}\right)$ is either 0 if $\operatorname{char}(\mathbb{F}) \nmid m$, or it has dimension 1 if $\operatorname{char}(\mathbb{F}) \mid m$ (see [5, Example A42]). Hence Theorem 2.7 gives the results.

Remark 4.2. As mentioned in the Introduction, the fact that $\operatorname{Der}(\varepsilon)$ is 0 for any evolution algebra $\mathcal{E}$ with $\mathcal{E}^{2}=\mathcal{E}$ over $\mathbb{C}$ has already been proved in 2 , Theorem 2.1].

Consider the algebra of dual numbers $\mathbb{F}[\epsilon]=\mathbb{F} 1 \oplus \mathbb{F} \epsilon$, with $\epsilon^{2}=0$, and the natural homomorphism $\pi: \mathbb{F}[\epsilon] \longrightarrow \mathbb{F}$ in $\operatorname{Alg}_{\mathbb{F}}(\pi(1)=1, \pi(\epsilon)=0)$. Given a graph $\Gamma=(V, E)$, Lie $(\operatorname{Diag}(\Gamma))$ is the kernel of the induced group homomorphism $\pi_{*}: \operatorname{Diag}(\Gamma)(\mathbb{F}[\epsilon]) \longrightarrow \operatorname{Diag}(\Gamma)(\mathbb{F})$. The elements of ker $\pi_{*}$ are the maps

$$
\begin{aligned}
\varphi: V & \longrightarrow \mathbb{F}[\mathcal{E}] \\
v & \mapsto 1+\delta(v) \epsilon,
\end{aligned}
$$

for a linear map $\delta: V \longrightarrow \mathbb{F}$, such that, for any $(v, w) \in E, \varphi(w)=\varphi(v)^{2}$, which is equivalent to $\delta(w)=2 \delta(v)$.

Therefore we obtain the following straightforward consequence of Theorems 4.1, 2.7 and 2.9,

Corollary 4.3. Let $\mathcal{E}$ be an evolution algebra with $\mathcal{E}^{2}=\mathcal{E}$ over a field $\mathbb{F}$ of characteristic $p \neq 0,2$. Let $B$ be a natural basis and let $\Gamma=\Gamma(\mathcal{E}, B)$ be the associated graph. Let $\Gamma_{i}=\left(V_{i}, E_{i}\right)\left(V_{i} \subseteq B\right), i=1, \cdots, r$, be the connected components of $\Gamma$ such that $p \mid 2^{\mathrm{b}\left(\Gamma_{i}\right)}-1$. For any $i=1, \ldots, r$, fix an element $v_{i} \in V_{i}$. Then a basis of $\operatorname{Lie}(\operatorname{Diag}(\Gamma))$ is given by $\hat{\delta}_{1}, \cdots, \hat{\delta}_{r}$, where

- $\hat{\delta}_{i}(v)=0$ if $v \notin V_{i}$,

- $\hat{\delta}_{i}\left(v_{i}\right)=v_{i}$

- $\hat{\delta}_{i}(w)=2^{\mathrm{b}(\gamma)} w$ if $w \in V_{i}$ and $\gamma=\left(w_{0}, e_{1}, w_{1}, \ldots, e_{n}, w_{n}\right)$ is a path connecting $w_{0}=v_{i}$ and $w_{n}=w$.

Example 4.4. The evolution algebra $\mathcal{E}$ in Example 3.3 has trivial group scheme of automorphisms, so $\operatorname{Der}(\mathcal{E})=0$ for any ground field $\mathbb{F}$.

However, for the evolution algebra $\mathcal{E}$ in Example 3.5, we have the short exact sequence in (10), and $\operatorname{Diag}(\Gamma) \simeq \boldsymbol{\mu}_{3}$. Hence $\operatorname{Der}(\mathcal{E})=0$ unless $\operatorname{char}(\mathbb{F})=3$. In the later case, $\operatorname{Der}(\mathcal{E})$ is spanned by the map $d: v_{1} \mapsto v_{1}$, $v_{2} \mapsto 2 v_{2}=-v_{2}$.

Remark 4.5. It must be remarked that for $\alpha=1$, the evolution algebra $\mathcal{E}$ in Example 3.5 is the two-dimensional split para-Hurwitz algebra, and hence, for arbitrary $\alpha(\neq 0), \mathcal{E}$ is a symmetric composition algebra (see [4] and references therein).

As shown in Example 3.5, the short exact sequence

$$
1 \longrightarrow \boldsymbol{\mu}_{3} \longrightarrow \operatorname{Aut}(\mathcal{E}) \longrightarrow \mathrm{C}_{2} \longrightarrow 1
$$

splits if and only if $\alpha \in \mathbb{F}^{3}$, that is, if and only if $\mathcal{E}$ is, up to isomorphism, the split two-dimensional para-Hurwitz algebra. 


\section{REFERENCES}

[1] Y. Cabrera Casado, M. Siles Molina, and M.V. Velasco, Evolution algebras of arbitrary dimension and their decompositions, Linear Algebra Appl. 495 (2016), 122-162.

[2] L.M. Camacho, J.R. Gómez, B.A. Omirov, and R.M. Turdibaev, The derivations of some evolution algebras, Linear Multilinear Algebra 61 (2013), no. 3, 309-322.

[3] M.I. Cardoso-Gonçalves, D. Martín-Barquero, C. Martín-González, and M. SilesMolina, Squares and associative representations of two-dimensional evolution algebras, preprint arXiv:1807.02362.

[4] A. Elduque, Symmetric composition algebras, J. Algebra 196 (1997), no. 1, 282-300.

[5] A. Elduque and M. Kochetov Gradings on simple Lie algebras, Mathematical Surveys and Monographs, vol. 189, American Mathematical Society, Providence, RI, 2013.

[6] A. Elduque and A. Labra, Evolution algebras and graphs. J. Algebra Appl. 14 (2015), no. $7,1550103,10 \mathrm{pp}$.

[7] A. Elduque and A. Labra, On nilpotent evolution algebras, Linear Algebra Appl. 505 (2016), 11-31.

[8] J.S. Milne, Algebraic groups, Cambridge Studies in Advanced Mathematics, vol. 170, Cambridge University Press, Cambridge, 2017.

[9] J.P. Tian, Evolution algebras and their applications, Lecture Notes in Mathematics, vol. 1921, Springer-Verlag, Berlin, 2008.

[10] J.P. Tian and P. Vojtechovsky, Mathematical concepts of evolution algebras in nonMendelian genetics, Quasigroups Related Systems 14 (2006) (1), 111-122.

[11] W.C. Waterhouse, Introduction to affine group schemes, Graduate Texts in Mathematics, vol. 66, Springer-Verlag, New York-Berlin, 1979.

Departamento de Matemáticas e Instituto Universitario de Matemáticas y Aplicaciones, Universidad de Zaragoza, 50009 Zaragoza, Spain

E-mail address: elduque@unizar.es

Departamento de Matemáticas, Facultad de Ciencias, Universidad de Chile. Casilla 653, Santiago, Chile

E-mail address: alimat@uchile.cl 